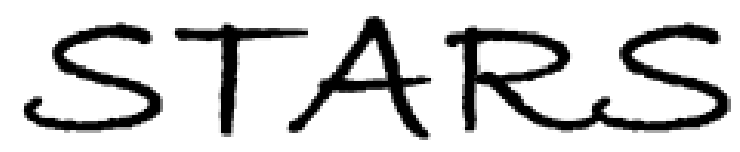

University of Central Florida

STARS

$1-1-2006$

\title{
Scanless ultraviolet remote sensor for limb profile measurements from low earth orbit
}

\author{
Andrey Krywonos \\ University of Central Florida \\ James E. Harvey \\ University of Central Florida \\ Robert E. Daniell \\ Nicolas Parent \\ University of Central Florida \\ Richard Eastes \\ University of Central Florida
}

Find similar works at: https://stars.library.ucf.edu/facultybib2000

University of Central Florida Libraries http://library.ucf.edu

This Article; Proceedings Paper is brought to you for free and open access by the Faculty Bibliography at STARS. It has been accepted for inclusion in Faculty Bibliography 2000s by an authorized administrator of STARS. For more information, please contact STARS@ucf.edu.

\section{Recommended Citation}

Krywonos, Andrey; Harvey, James E.; Daniell, Robert E.; Parent, Nicolas; and Eastes, Richard, "Scanless ultraviolet remote sensor for limb profile measurements from low earth orbit" (2006). Faculty Bibliography 2000s. 6318.

https://stars.library.ucf.edu/facultybib2000/6318

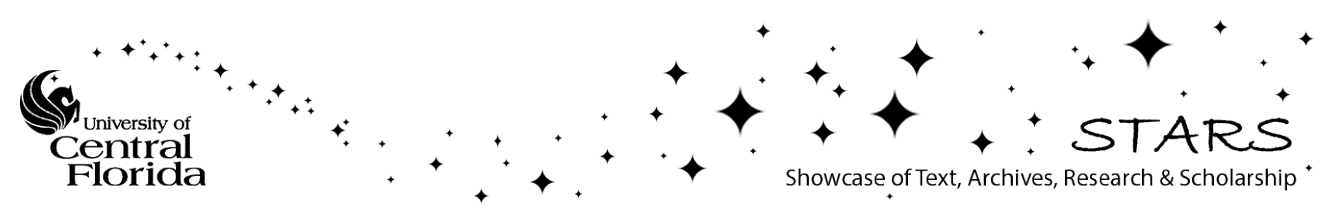




\section{Scanless ultraviolet remote sensor for limb profile measurements from low Earth orbit}

Andrey Krywonos, James E. Harvey, Robert E. Daniell Jr., Nicolas Parent, Richard W. Eastes

Andrey Krywonos, James E. Harvey, Robert E. Daniell Jr., Nicolas Parent, Richard W. Eastes, "Scanless ultraviolet remote sensor for limb profile measurements from low Earth orbit," Proc. SPIE 5660, Instruments, Science, and Methods for Geospace and Planetary Remote Sensing, (30 December 2004); doi: 10.1117/12.580861

Event: Fourth International Asia-Pacific Environmental Remote Sensing Symposium 2004: Remote Sensing of the Atmosphere, Ocean, Environment, and Space, 2004, Honolulu, Hawai'i, United States 


\title{
Scanless Ultraviolet Remote Sensor for Limb Profile Measurements from Low Earth Orbit
}

\author{
Andrey Krywonos and James E. Harvey \\ Center for Research and Education in Optics and Lasers (CREOL) \\ The University of Central Florida, Orlando, Florida 32816 \\ Robert E. Daniell \\ Computational Physics Inc. (CPI) \\ 207 Fulton Street, Norwood, Mass., 02062-2707 \\ Nicolas Parent and Richard Eastes \\ Florida Space Institute (FSI) \\ The University of Central Florida, Orlando, Florida 32826
}

\begin{abstract}
Ultraviolet (UV) observations are essential for meeting operational requirements for space weather specification. Such observations provide valuable information about neutral and ion density variations in the Earth's upper atmosphere. However, the resources required to support the necessary ultraviolet sensors are significant. Current operational sensors measure the limb profiles by mechanically scanning the field-of-view across the limb. This mechanical scan mechanism requires significant power, has a potential for failure, and the high counting rates during observations near the peak of the limb profile require high speed detectors to accommodate the counting rates when using the high sensitivity sensors. Also, the attitude information and stability needed for accurate limb profiles are more difficult on smaller spacecraft and require considerable resources. This paper describes an instrument that can provide limb observations of the UV airglow by aligning the slit perpendicular to the limb. To measure the limb profile without scanning requires a combination of wide field-of-view and high spatial resolution which previous instruments have been unable to provide. This approach would require significantly less resources (power, weight, etc.) than current sensors, while providing similar performance.
\end{abstract}

Keywords: Scanless UV Remote Sensor, Earth Limb Profile Measurements, UV Imaging Spectrometer

\subsection{INTRODUCTION}

Measurements of the atmospheric emission profile can be used to improve our understanding of the neutral and ion densities in the Earth's space environment. Observations are needed to validate research models and as input for operational models of the space environment. The need for such data has led to the flight of scientific and operational sensors for ultraviolet (UV) observations (for example: instruments on the DMSP and TIMED satellites).

In the daytime UV observations are sensitive to the relative and the absolute neutral densities. Both are important for space weather applications. Variations in the relative neutral density $\left(\mathrm{O} / \mathrm{N}_{2}\right)$ cause differences between observed and calculated ion densities ${ }^{1}$. These same changes in the neutral density ratio will produce changes in both the daytime and nighttime ionospheric densities. Recent work ${ }^{2,3}$ has shown large changes in the $\mathrm{O} / \mathrm{N}_{2}$ ratio due to storm time effects, which are consistent with the analysis of daytime UV measurements ${ }^{4}$. Understanding changes in the neutral density is important for understanding the Earth's upper atmosphere.

At night the measured brightnesses depend directly on the ionospheric densities. Density depletions in the equatorial region, where degradation or loss of radio frequency signals can occur, are observable. 
Therefore, UV observations are potentially valuable for efforts to predict or specify outages in communications or navigation systems.

Current UV sensors, such as those used on the DMSP satellite, measure the emission profiles by mechanically scanning the field-of-view (FOV) across the limb. The mechanical scan mechanisms require significant power, are a source of substantial risk, and the high counting rates during observations near the peak of the limb profile require high speed detectors to accommodate the counting rates when using high sensitivity sensors. Also, the attitude information and stability needed for accurate limb profiles are greater than would be necessary when using a system without a mechanical scanning mechanism.

To measure the limb profile without scanning requires a combination of wide FOV and high spatial resolution which previous instruments have been unable to provide. This approach would require significantly fewer resources (power, weight, etc.) than current sensors, while providing equal or better performance. From a low Earth orbit such observations would complement the currently scheduled UV imagers and potential UV imagers at geostationary orbit. The instrument discussed in this paper, HIDEN (Height Information for Densities of Electrons and Neutrals), can provide limb observations of the UV airglow by aligning the slit perpendicular to the limb. The limb viewing geometry from low Earth orbit is illustrated in Figure 1; however, the instrument could readily operate in a higher orbit. The performance requirements and goals for the HIDEN instrument are listed in Table 1.

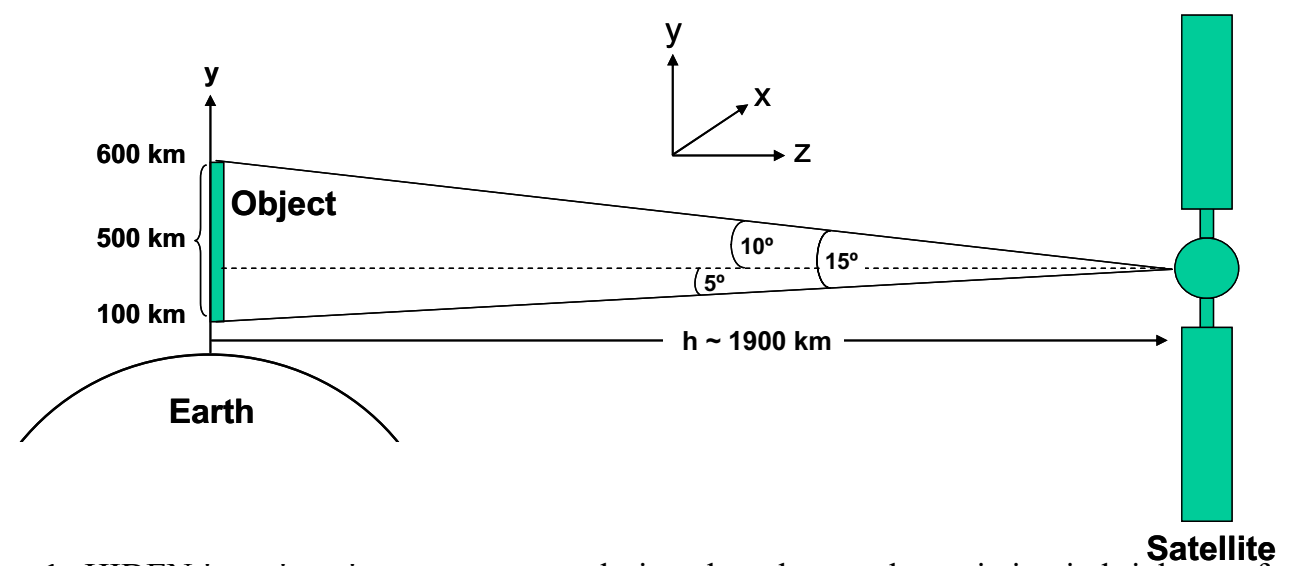

Figure 1. HIDEN is an imaging spectrometer designed to observe the variation in brightness from the Earth's limb (the object) at altitudes between 100 and $600 \mathrm{~km}$. The object is imaged in the $y$-direction and spectrally dispersed by a diffraction grating in the $\mathrm{x}$-direction.

Table 1: HIDEN Performance Requirements and Goals.

\begin{tabular}{|l|c|c|}
\hline Instrument Parameter & Requirement & Goal \\
\hline Instrument Length: & $<300 \mathrm{~mm}$ & $<250 \mathrm{~mm}$ \\
Entrance Pupil Size: & $15 \mathrm{~mm} \mathrm{X} 15 \mathrm{~mm}$ & $20 \mathrm{~mm}$ X $20 \mathrm{~mm}$ \\
Detector Diameter (active area): & $50 \mathrm{~mm}$ & $37.5 \mathrm{~mm}$ \\
Field-of-View: & 15 degrees & 15 degrees \\
Spatial (angular) Resolution: & $10 \mathrm{~km}(0.3 \mathrm{deg})$ & $7 \mathrm{~km}(0.2 \mathrm{deg}$ \\
Spectral Range: & $130 \mathrm{~nm}-170 \mathrm{~nm}$ & $130 \mathrm{~nm}-170 \mathrm{~nm}$ \\
Spectral Resolution: & $0.4 \mathrm{~nm}$ & $0.3 \mathrm{~nm}$ \\
\hline
\end{tabular}




\subsection{PREVIOUS SPECTROGRAPHIC IMAGERS}

The UV spectrographic imagers on recent scientific and operational satellites have been in orbits with altitudes of $625-850 \mathrm{~km}$. Lower orbits may not be above the peak of the nighttime $F$ region, and measurements of this region have been one of the motivations for flying the instruments. Higher orbits would be above those used for recent operational satellites (e.g. the DMSP satellites). Therefore, orbits with altitudes of $\sim 840 \mathrm{~km}$ are likely to be used for future flights since changing the altitude significantly the impacts some operational sensors.

Two types of spectrographic imagers have been flown on recent missions. Instruments such as the Global Ultraviolet Imager (GUVI) on the NASA TIMED (Thermosphere Ionosphere Mesosphere Energy and Dynamics) satellite and the Special Sensor Ultraviolet Spectrographic Imager (SSUSI) on the DMSP satellite observe the Earth's limb and disk. ${ }^{5,6}$ Such instruments emphasize providing information across the path of the satellite. Another type of instrument, such as the Low-Resolution Airglow and Aurora Spectrograph (LORAAS) on the Advanced Research and Global Observation Satellite (ARGOS) or the Special Sensor Ultraviolet Limb Imager (SSULI) on the DMSP satellite, emphasize providing limb observations along the track of the satellite. ${ }^{7}$ Additional details for these instruments are given in Table 2. Other UV spectrographic imagers, e.g. the Remote Atmospheric and Ionospheric Detection System (RAIDS), have similar spatial and spectral resolution capabilities. ${ }^{8}$

From an altitude of $840 \mathrm{~km}$, a FOV of approximately 15 degrees is needed to provide the necessary coverage of the limb. All of the instruments discussed have significantly smaller FOVs which are scanned mechanically to provide observations of the limb. While the SSUSI FOV is $\sim 12$ degrees in one dimension, the spatial resolution along the slit is not sufficient for the desired limb observations.

Table 2. Characteristics of previous UV spectrographic imagers.

\begin{tabular}{|c|c|c|c|c|}
\hline Instrument & $\begin{array}{l}\text { Field-of- } \\
\text { view }\end{array}$ & $\begin{array}{c}\text { Aperture } \\
\left(\mathrm{mm}^{2}\right)\end{array}$ & Spatial Resolution & $\begin{array}{c}\text { Wavelength Range \& } \\
\text { Resolution }\end{array}$ \\
\hline $\begin{array}{l}\text { LORAAS } \\
\text { (or SSULI) }\end{array}$ & $0.15^{\circ} \times 2.4^{\circ}$ & $70 \times 70$ & $\begin{array}{c}5 \mathrm{~km} \text { vertical (on limb) } \\
7 \mathrm{~km} \times 7 \mathrm{~km}\end{array}$ & $80-170 \mathrm{~nm}$ at $1.1-1.8 \mathrm{~nm}$ \\
\hline $\begin{array}{c}\text { GUVI } \\
\text { (or SSUSI) }\end{array}$ & $0.8^{\circ} \times 11.8^{\circ}$ & $20 \times 20$ & $\begin{array}{c}\text { (nadir viewing@ } 140 \\
\text { km) }\end{array}$ & $\begin{array}{c}115-180 \mathrm{~nm} \text { at } 1.2,1.9 \text { or } \\
4.2 \mathrm{~nm}\end{array}$ \\
\hline $\begin{array}{c}\text { RAIDS } \\
\text { (FUV) }\end{array}$ & $0.1^{\circ} \times 4.0^{\circ}$ & $21 \times 25$ & $3 \mathrm{~km}$ vertical (on limb) & $130-170 \mathrm{~nm}$ at $0.7 \mathrm{~nm}$ \\
\hline
\end{tabular}

\subsection{CONCEPTUAL DESIGN AND OPTICAL PERFORMANCE CRITERIA}

The current GUVI and SSUSI instruments ${ }^{5,6}$ are two-element designs. A two-element design was thus considered first. This system used a telescope mirror to collect and focus the UV radiation on the spectrograph slit, and a concave grating to disperse the radiation and re-image the slit onto the detector. The entrance pupil (aperture stop) was imaged onto the grating by the telescope mirror, and the slit was imaged onto the detector by the concave grating. The two element design was unable to satisfy the performance requirements even with a $15 \mathrm{~mm} \mathrm{X} 15 \mathrm{~mm}$ aperture and aspheric toroidal (bi-conic) surfaces on both the telescope mirror and the concave grating.

A three-element conceptual design consisting of a telescope mirror which collects the UV radiation and focuses it upon the spectrograph slit, a collimating mirror, and a concave grating to disperse the radiation and re-image the slit onto the detector was then evaluated. The basic configuration is 
illustrated in Figure 2. This configuration provides additional design variables and results in a larger system focal ratio, both of which help to reduce aberrations and improve system performance. A long sunshade is also incorporated into the design.

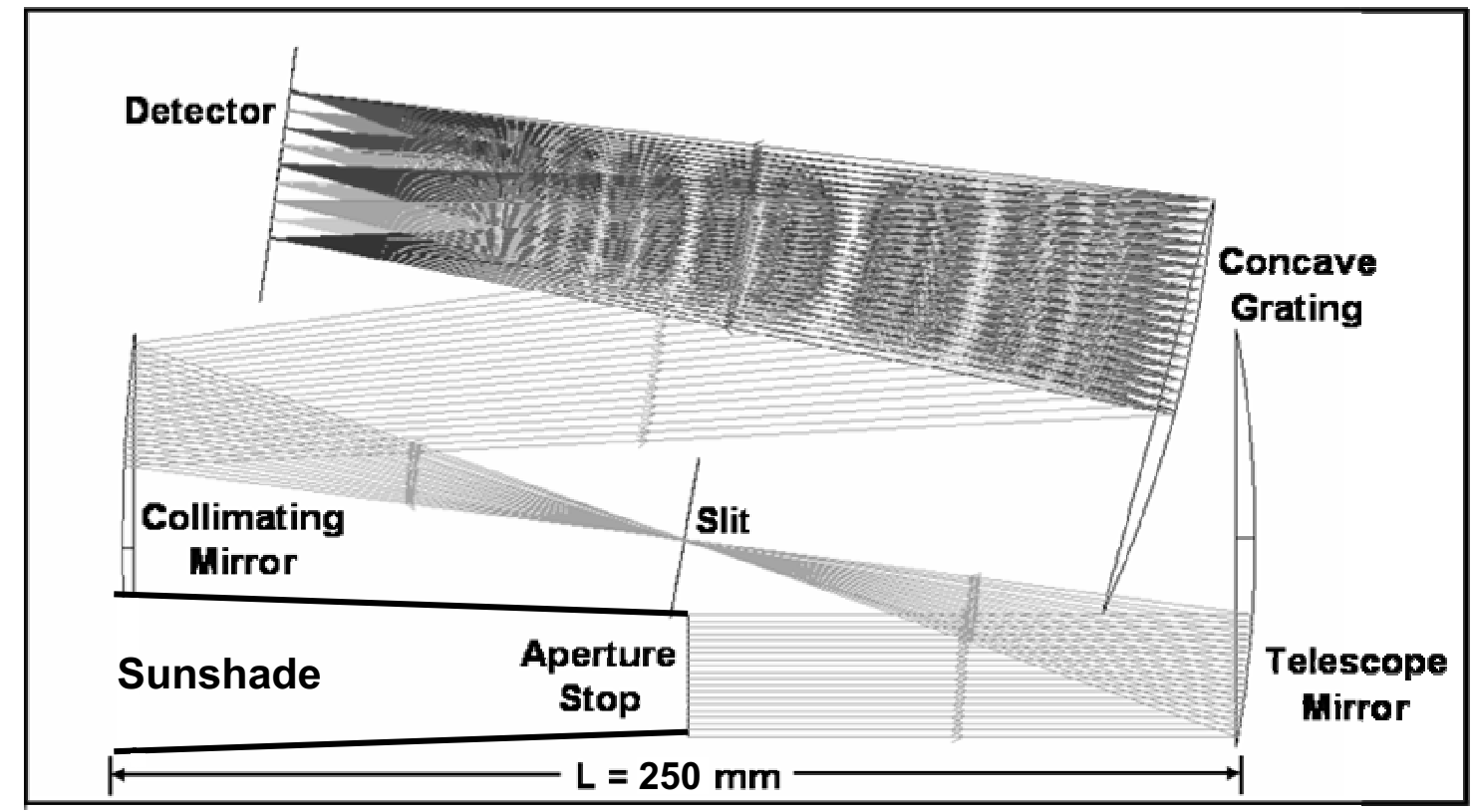

Figure 2. Three-element conceptual design consisting of a telescope mirror which collects the UV radiation and focuses it upon the spectrograph slit, a collimating mirror, and a concave grating to disperse the radiation and re-image the slit onto the detector.

In order to proceed with detailed quantitative performance evaluation and design evolution, we defined the spatial (angular) resolution as the angular separation of two point sources such that their separation in the detector plane is equal to the width of a one-dimensional rectangle (in the spatial dimension) containing $70 \%$ of the energy in one of the images. Similarly, we defined the spectral resolution as the difference between two wavelengths, $\Delta \lambda$, such that the images of a point source at those two wavelengths would be separated on the detector by an amount equal to the width of a onedimensional rectangle (in the spectral dimension) containing $70 \%$ of the energy of one of the images.

\subsection{PERFORMANCE ANALYSIS AND DESIGN EVOLUTION}

The Zemax optical design code was used for performance evaluation and design evolution. The tilt angles of the reflective components were reduced as much as mechanical constraints (e.g., mounting of the detector and entrance slit) would allow in order to minimize aberrations. We eliminated bi-conic surfaces as this would permit fabrication techniques resulting in smoother surfaces and less scattering at the ultraviolet wavelengths of interest. The design was optimized to balance the spatial and spectral resolution in a way that would allow both requirements to be met. We evaluated both traditional ruled and custom variable-line-spaced (VLS) gratings, the latter of which were designed in Zemax as part of the optimization process. Our three-element preliminary design is illustrated in Figure 3. This design utilizes a toroidal concave grating, and the corresponding system and individual component parameters are listed in Table 3. A second design utilizing a spherical VLS grating has the same basic layout, and its system and individual component parameters are listed in Table 4. 


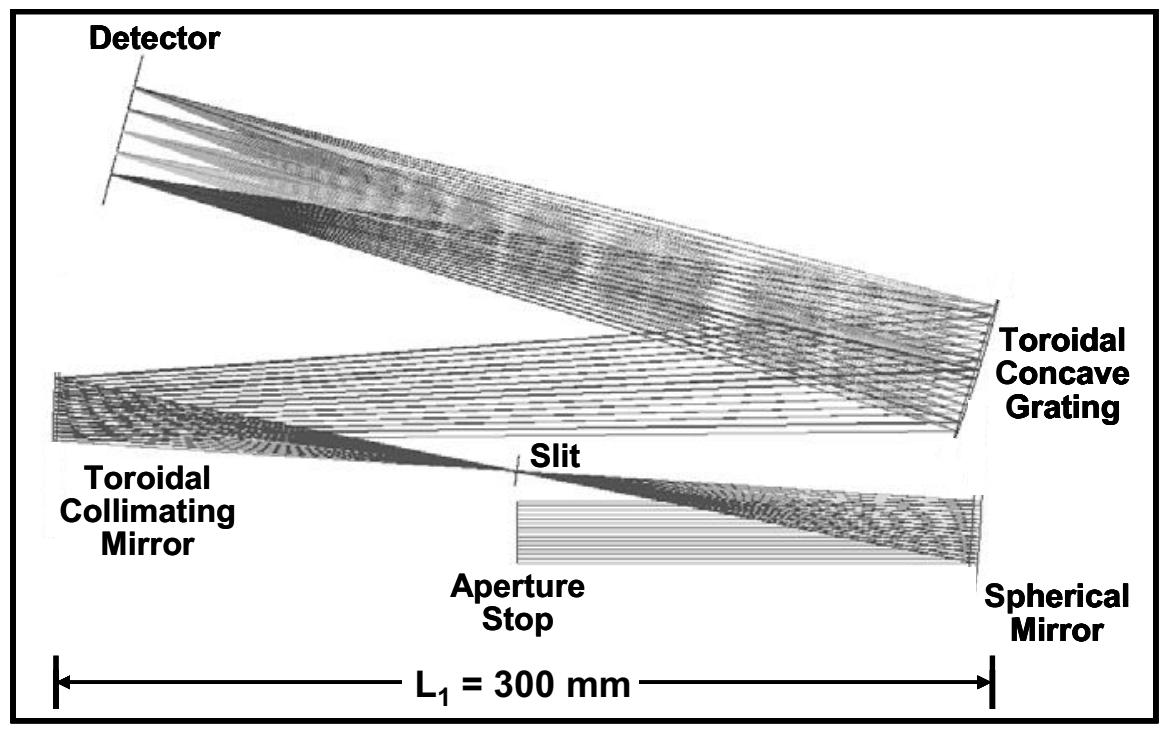

Figure 3. Three-element preliminary design consisting of a spherical telescope mirror which collects the UV radiation and focuses it upon the spectrograph slit, a toroidal collimating mirror, and a toroidal concave grating to disperse the radiation and re-image the slit onto the detector.

Table 3. Spectrograph System and Component Parameters (toroidal grating).

\begin{tabular}{|ll|}
\hline Spectrograph System Parameters & Telescope Mirror (Sphere) \\
Entrance Pupil: $20 \mathrm{~mm}$ X $20 \mathrm{~mm}$ & Dimensions: $21 \mathrm{~mm} \mathrm{X} 60 \mathrm{~mm}$ \\
Detector: $50 \mathrm{~mm}$ diameter (active area) & Radius of Curvature: $\sim 300 \mathrm{~mm}$ \\
Slit: Width $=0.25 \mathrm{~mm}$, Length $=39.8 \mathrm{~mm}$ & \\
Total System Length: $300 \mathrm{~mm}$ & Concave Grating (Toroid) \\
System Field-of-View: $15^{\circ}\left(+5^{\circ}\right.$ to $\left.-10^{\circ}\right)$ & Dimensions: $43 \mathrm{~mm}$ X $40 \mathrm{~mm}$ \\
Spectrograph Collimating Mirror (Toroid) & Radii of Curvature: $\mathrm{R}_{\mathrm{x}} \sim 380 \mathrm{~mm}, \mathrm{R}_{\mathrm{y}} \sim 390 \mathrm{~mm}$ \\
Dimensions: $22 \mathrm{~mm} \mathrm{X} 61 \mathrm{~mm}$ & Grating Period: 2500 lines $/ \mathrm{mm}$ \\
Radii of Curvature: $\mathrm{Rx} \sim 590 \mathrm{~mm}, \mathrm{Ry} \sim 610 \mathrm{~mm}$ & \\
\hline
\end{tabular}

Table 4. Spectrograph System and Component Parameters (VLS grating).

\begin{tabular}{|ll|}
\hline Spectrograph System Parameters & Telescope Mirror (Sphere) \\
Entrance Pupil: $20 \mathrm{~mm}$ X $20 \mathrm{~mm}$ & Dimensions: $21 \mathrm{~mm}$ X $60 \mathrm{~mm}$ \\
$\begin{array}{l}\text { Detector: } 50 \mathrm{~mm} \text { diameter (active area) } \\
\text { Slit: Width }=0.25 \mathrm{~mm} \text {, Length }=39.8 \mathrm{~mm}\end{array}$ & Radius of Curvature: $\sim 300 \mathrm{~mm}$ \\
Total System Length: $300 \mathrm{~mm}$ & \\
System Field-of-View: $15^{\circ}\left(+5^{\circ}\right.$ to $\left.-10^{\circ}\right)$ & Concave Grating (Sphere) \\
& Dimensions: $43 \mathrm{~mm}$ X $40 \mathrm{~mm}$ \\
Spectrograph Collimating Mirror (Toroid) & Radius of Curvature: $\sim 370 \mathrm{~mm}$ \\
Dimensions: $22 \mathrm{~mm} \mathrm{X} 61 \mathrm{~mm}$ & Grating Period: 2530 lines $/ \mathrm{mm}$ \\
Radii of Curvature: $\mathrm{Rx} \sim 590 \mathrm{~mm}, \mathrm{Ry} \sim 610 \mathrm{~mm}$ & \\
\hline
\end{tabular}

\subsection{OPTICAL PERFORMANCE PREDICTIONS OF PRELIMINARY DESIGN}

The resolution calculations were performed using a combination of Zemax and Matlab. For each wavelength and field angle, a geometric image analysis was done and the resulting ray density (spot diagram) data was saved, imported into Matlab, and converted into a geometric point spread function 
(density of ray intercepts). A convolution with the image of the slit in the detector plane was then performed to produce the geometric point spread function on the detector which included the effect of the slit. The spatial and spectral resolutions were then calculated for various wavelengths and field angles using our definitions as described in section 3.0.

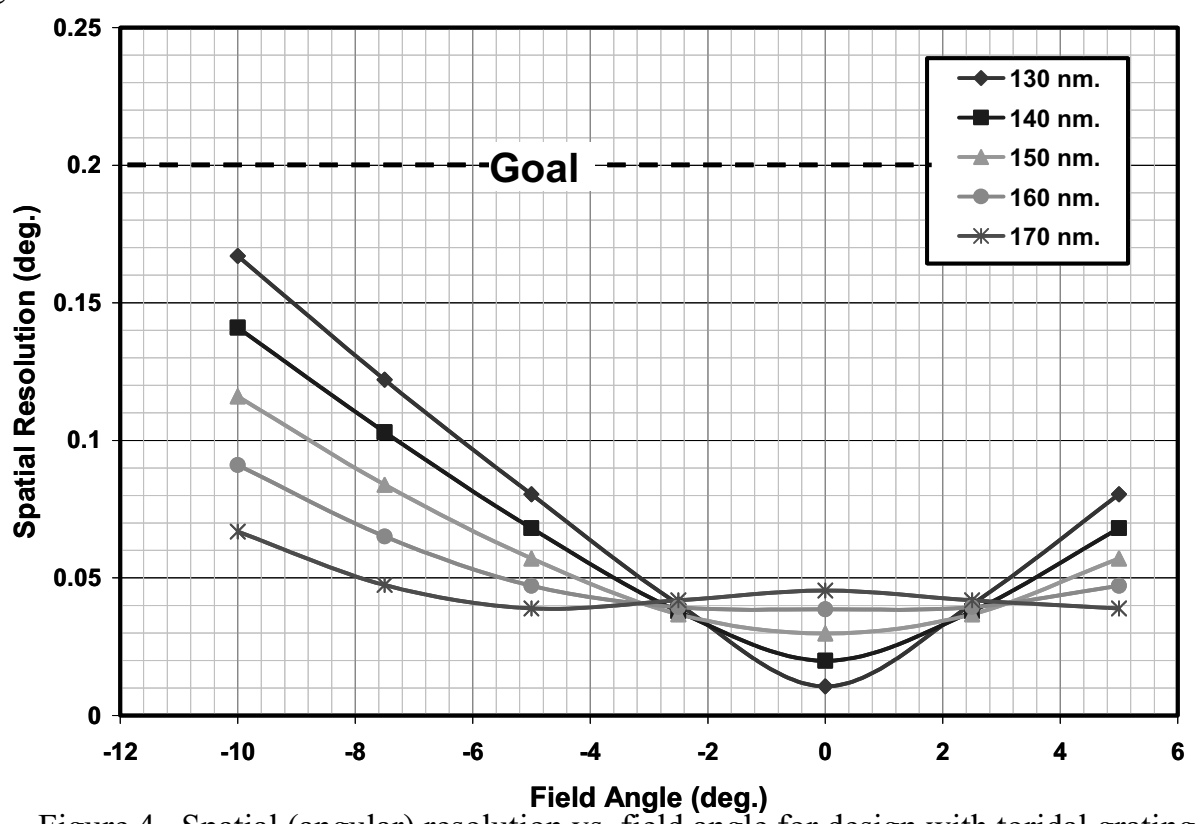

Figure 4. Spatial (angular) resolution vs. field angle for design with toridal grating.

Figures 4 and 5 show the spatial and spectral resolution respectively for our preliminary design which utilizes a traditional toroidal grating. The spatial resolution results meet the goal for all wavelengths across the entire FOV. The spectral resolution results meet the goal for much of the FOV, and meet the requirement except near the edge of the FOV for wavelengths below $160 \mathrm{~nm}$.

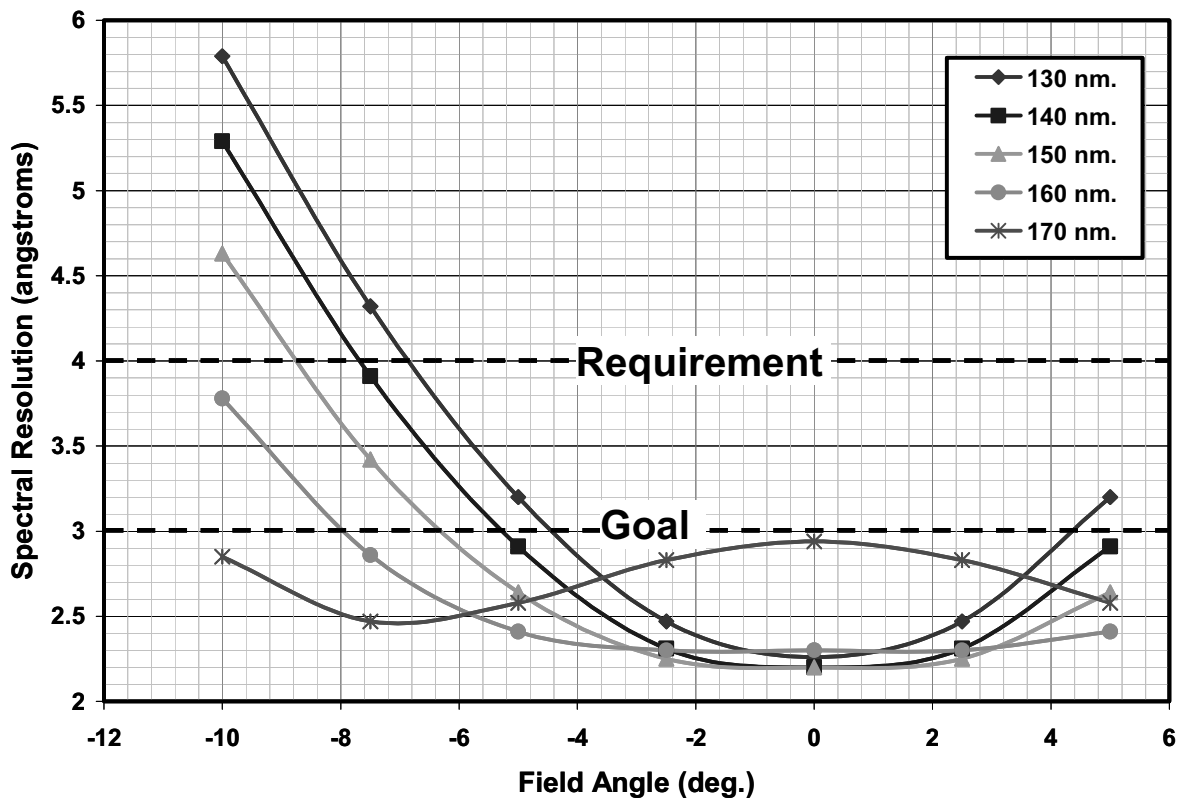

Figure 5. Spectral resolution vs. field angle for design with toridal grating. 
Figures 6 and 7 show the spatial and spectral resolution respectively for our preliminary design which utilizes a spherical VLS grating. The results are similar to the first design. The spatial resolution results again meet the goal for all wavelengths across the entire FOV. Similarly, the spectral resolution results meet the goal over much of the FOV, and meet the requirement except near the edge of the FOV for wavelengths below $160 \mathrm{~nm}$.

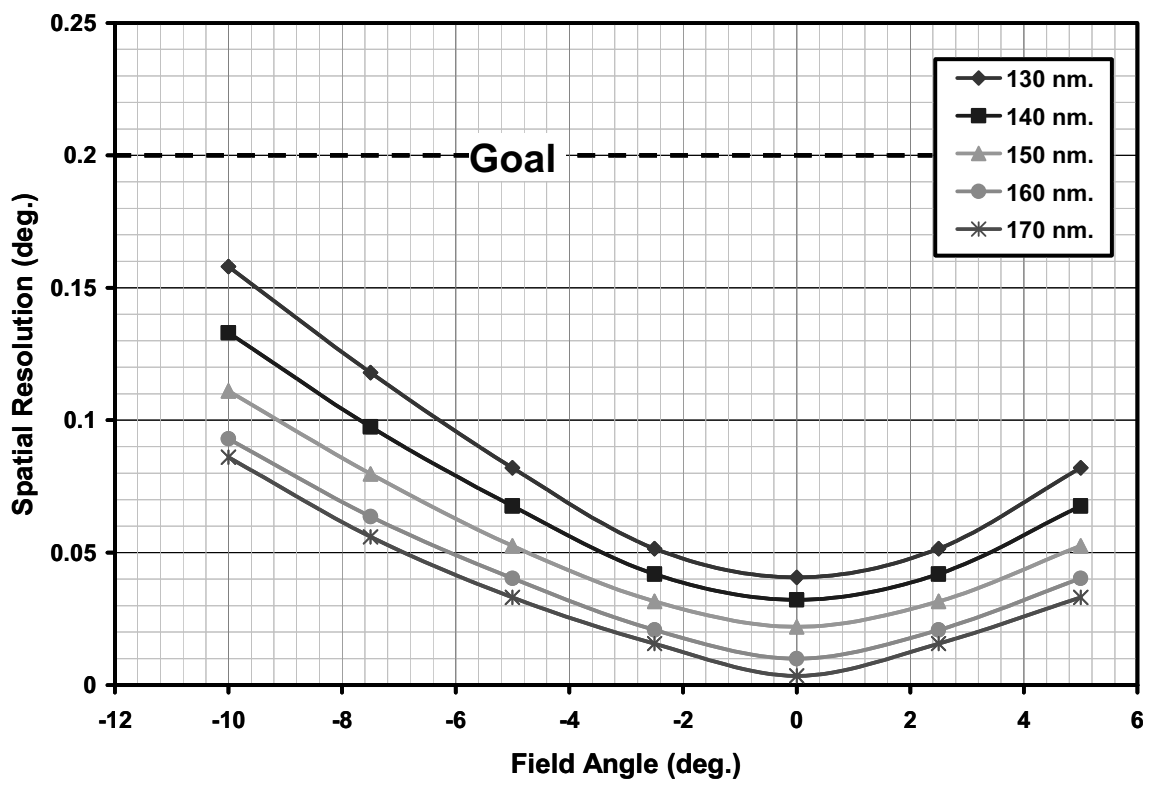

Figure 6. Spatial (angular) resolution vs. field angle for design with spherical VLS grating.

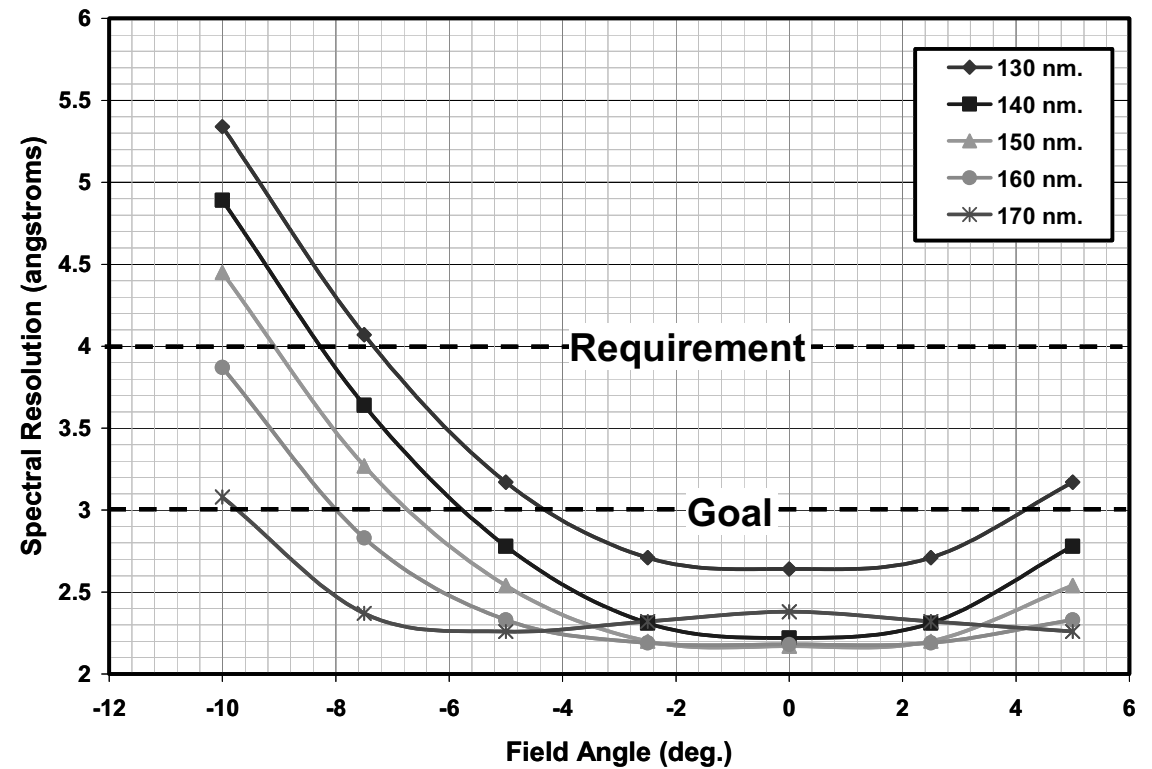

Figure 7. Spectral resolution vs. field angle for design with spherical VLS grating.

The results for the design utilizing a VLS grating do not represent a significant increase over those of the design with a traditional toroidal grating. However since the VLS grating is spherical, its surface can 
be made smoother than that of the toroidal grating, and would produce less scatter from surface roughness.

\subsection{MODELED MEASUREMENTS}

To provide a better understanding of the measurements from the instrument described in the previous sections, calculated signals for some of the commonly measured emissions are shown in Figures 8-10. These plots show limb profiles (in counts/s) of airglow emissions from atomic oxygen (130.4 and 135.6 $\mathrm{nm}$ ) and molecular nitrogen (Lyman-Birge-Hopfield, LBH, bands) in the daytime, and from atomic oxygen $(135.6 \mathrm{~nm})$ at night. The LBH short band includes 140-150 nm emissions from molecular nitrogen and the LBH long band includes $160-170 \mathrm{~nm}$ emissions. The conditions are equinox, low solar activity $(\mathrm{F} 10.7=100)$, and low geomagnetic activity $(\mathrm{Ap}=0)$. The satellite was at $400 \mathrm{~km}$ altitude at $\left(35^{\circ} \mathrm{N}, 70^{\circ} \mathrm{W}\right)$ and the instrument was looking at an azimuth of $285^{\circ}$ (that is, $15^{\circ}$ north of due west). The central pixel of the slit was oriented toward a zenith angle of $101^{\circ}$. Under these conditions, tangent altitudes of $387 \mathrm{~km}$ to $50 \mathrm{~km}$, are observed.

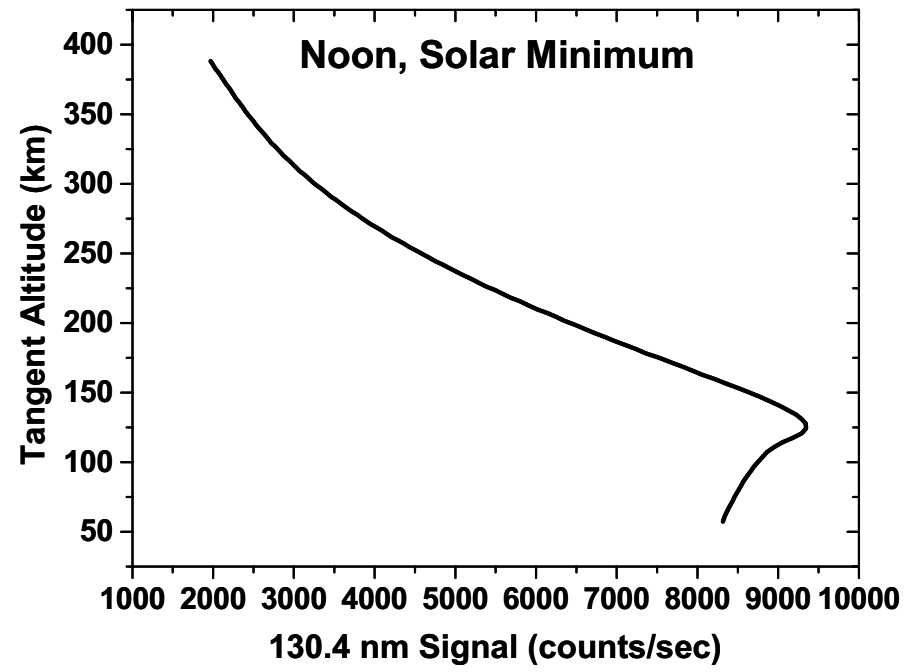

Figure 8. Atomic oxygen $130.4 \mathrm{~nm}$ counting rates near noon during solar minimum.

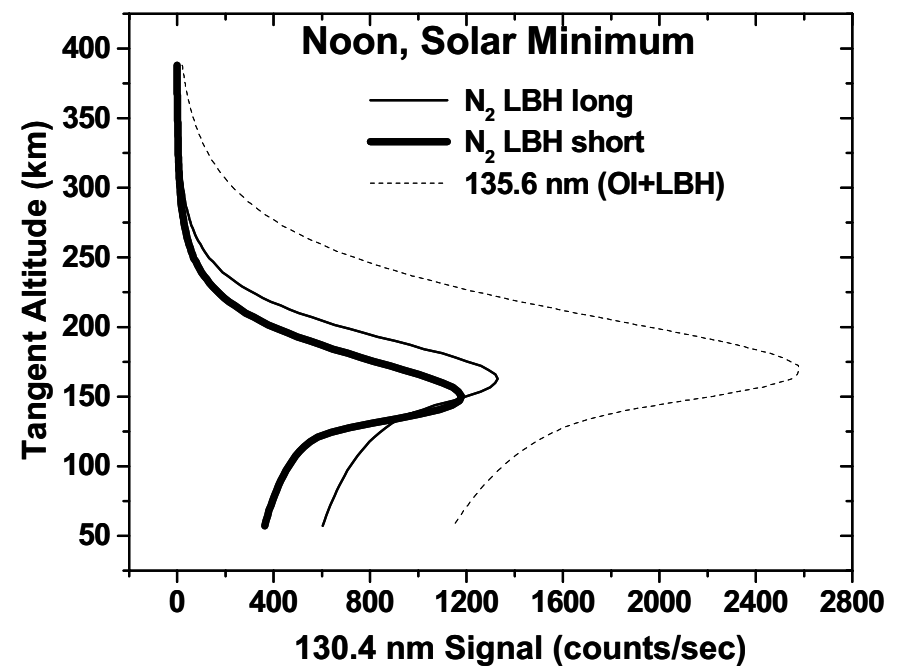

Figure 9. Atomic oxygen $135.6 \mathrm{~nm}(\mathrm{OI})$ and $\mathrm{N}_{2}$ Lyman-Birge-Hopfield (LBH) band counting rates near noon during solar minimum. 


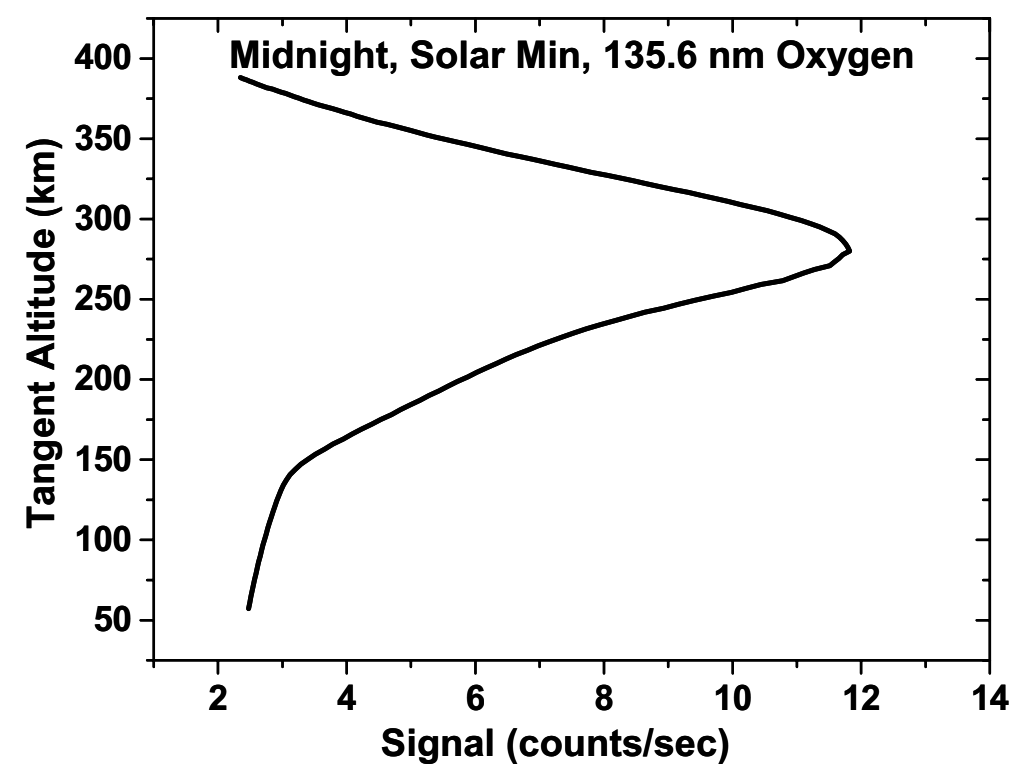

Figure 10. Atomic oxygen $135.6 \mathrm{~nm}$ from the equator near midnight during solar minimum

The count rates are based on a reflection efficiency of 0.85 for each mirror, a grating efficiency of 0.3 , a detector with a peak efficiency of $25 \%$ (falling to $10 \%$ at the longest wavelength), and a slit width of $0.25 \mathrm{~mm}$.

\subsection{SUMMARY}

A scanless imaging spectrometer for providing height information of densities of electrons and neutrals (HIDEN) as a function of altitude in the Earth's limb has been described. A three-element preliminary design which is capable of achieving a spatial (angular) resolution of less than 0.2 degrees over a 15 degree FOV and a spectral resolution range of $130 \mathrm{~nm}<\lambda<170 \mathrm{~nm}$ has been achieved. Detailed performance evaluation curves indicate that a spectral resolution of less than $0.3 \mathrm{~nm}$ is achieved over the entire spectral range of interest for field angles -5 degrees $<\theta<5$ degrees, and less than $0.4 \mathrm{~nm}$ over the remaining five degrees of the non-symmetrical 15 degree FOV (-10 degrees $<\theta<-5$ degrees) for wavelengths greater than $160 \mathrm{~nm}$.

The preliminary remote sensor design allows a $400 \mathrm{~mm}^{2}$ collecting aperture and can be packaged in a $300 \mathrm{~mm}$ long instrument which includes a $150 \mathrm{~mm}$ long sunshade for stray radiation control. Utilizing a variable-line-space (VLS) holographic grating should allow us to achieve the above performance with a spherical concave grating that will result in less surface scatter from these short ultraviolet wavelengths.

This scanless imaging spectrometer design will provide equal or better performance while requiring substantially less resources (power, weight, etc.) and operating more reliably than current designs with external scanning mirrors and their associated mechanisms.

The analysis shows that the necessary combination of wide field-of-view and high resolution needed to measure the limb profile without mechanical scanning is feasible in a relatively compact instrument design. This approach would require significantly less resources (power, weight, etc.) than current sensors, and is capable of better performance. 


\subsection{ACKNOWLEDGMENTS}

This work was supported by NASA grant number NAG512981 to the University of Central Florida from the Living with a Star program.

\subsection{REFERENCES}

1. R. R. Meier, R. R. Conway, D. E. Anderson Jr., P. D. Feldman, R. W. Eastes, E. P. Gentieu, and A. B. Christensen, "The ultraviolet dayglow at solar maximum 3. Photoelectron-excited emissions of N2 and O", J. Geophys. Res., 90, 6608, 1985.

2. T. Killeen, Y. Won, R. Niciejewski, A. Burns, "Upper thermosphere winds and temperatures in the geomagnetic polar cap: Solar cycle, geomagnetic activity, and interplanetary magnetic field dependencies", J. Geophys. Res., 100 (A11), 21327-21342, 1995.

3. D. J. Strickland, R. E. Daniell, and J. D. Craven, "Negative ionospheric storm coincident with DE-1 observed thermospheric disturbance on October 14, 1981", J. Geophys. Res, 106, 21,049, 2001.

4. D. J. Strickland, J. Bishop, J.S. Evans, T. Majeed, P.M. Shen, R.J. Cox, R. Link, and R.E. Huffman, "Atmospheric ultraviolet radiance integrated code (AURIC): Theory, software architecture, inputs and selected results", Jour. of Quant. Spect. and Rad. Transf., 62, 689-742, 1999.

5. A. B. Christensen, L. J. Paxton, S. Avery, J. Craven, G. Crowley, D. C. Humm, H. Kil, R. R. Meier, C.-I. Meng, D. Morrison, B. S. Ogorzalek, P. Straus, D. J. Strickland, R. M. Swenson, R. L. Walterscheid, B. Wolven, and Y. Zhang, "Initial observations with the Global Ultraviolet Imager (GUVI) in the NASA TIMED satellite mission”, J. Geophys. Res., 108 (A12), 1451, doi:10.1029/2003JA009918, 2003

6. L. J. Paxton, C. I. Meng, G. H. Fountain, B. S. Ogorzalek, E. H. Darlington, J. Goldsten, S. Geary, D. Kusnierkiewicz, S. C. Lee, and K. Peacock, "Special Sensor UV Spectrographic Imager (SSUSI): An instrument description", Instrum. Planet. Terr. Atmos. Remote Sens., 1745, 2-16, 1992.

7. R. P. McCoy, K. F. Dymond, G. G. Fritz, S. E. Thonnard, R. R. Meier, and P. A. Regeon, "Special Sensor Ultraviolet Limb Imager: an ionospheric and neutral density profiler for the Defense Meteorological Satellite Program satellites," Optical Engineering, 33, 423-429, 1994.

8. R. P. McCoy, R. R. Meier, K. D. Wolfram, J. M. Picone, S. E. Thonnard, G. G. Fritz, J. S. Morrill, D. A. Hardin, A. B. Christensen, D. C. Kayser, J. B. Pranke, P. R. Strauss, "Far-ultraviolet imaging spectrograph and scanning grating spectrometers for the Remote Atmospheric and Ionospheric Detection System", Optical Engineering, 33, 430-437, 1994. 